\title{
Colloidal Aluminum Antimonide Quantum Dots
}

\author{
Houman Bahmani Jalali, ${ }^{\dagger, \nabla} \nabla_{\odot}$ Sadra Sadeghi, ${ }^{\dagger, \nabla}{ }^{\circ}$ Mehmet Sahin, ${ }^{\| \odot}$ Hande Ozturk, ${ }^{\perp}$ \\ Cleva W. Ow-Yang, ${ }^{\#(-)}$ and Sedat Nizamoglu* $*,+, \S^{\circ}$
}

${ }^{\dagger}$ Graduate School of Biomedical Science and Engineering, ${ }^{\ddagger}$ Graduate School of Material Science and Engineering, ${ }^{\S}$ Department of Electrical and Electronics Engineering, Koç University, Istanbul 34450, Turkey

"Department of Materials Science and Nanotechnology Engineering, Abdullah Gul University, Kayseri 38080, Turkey

${ }^{\perp}$ Department of Mechanical Engineering, Ozyegin University, Istanbul 34794, Turkey

\#Department of Material Science and Nano Engineering, Sabanci University, Istanbul 34956, Turkey

\author{
Supporting Information
}

ABSTRACT: AlSb is a less studied member of the III-V semiconductor family, and herein, we report the colloidal synthesis of AlSb quantum dots (QDs) for the first time. Different sizes of colloidal AlSb QDs ( 5 to $9 \mathrm{~nm}$ ) were produced by the controlled reaction of $\mathrm{AlCl}_{3}$ and $\mathrm{Sb}\left[\mathrm{N}\left(\mathrm{Si}(\mathrm{Me})_{3}\right)_{2}\right]_{3}$ in the presence of superhydride. These colloidal $\mathrm{AlSb}$ quantum dots showed excitonic transitions in the UV-A region and a tunable bandedge emission (quantum yield of up to 18\%) in the blue spectral range. Among all III-V quantum dots, these quantum dots show the brightest core emission in the blue spectral region.
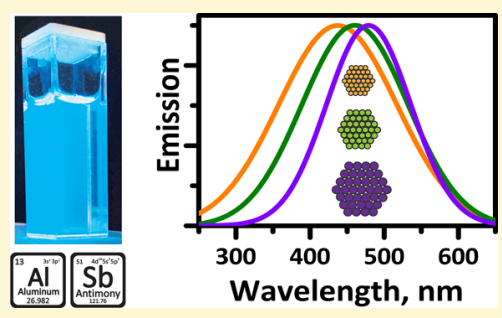

\section{INTRODUCTION}

Colloidal semiconductor quantum dots (QDs) opened up opportunities for low-temperature, low-cost, and large-area fabrication of optoelectronic and biological devices. ${ }^{1,2}$ Most popular and well-studied colloidal QDs contain $\mathrm{Cd}$ and $\mathrm{Pb}$, which are known to be highly toxic and carcinogenic for living systems. $^{3}$ As a solution, $\mathrm{Cd}$ - and $\mathrm{Pb}$-free III-V colloidal semiconductor QDs were introduced as nontoxic alternatives such as $\operatorname{InP}{ }^{4} \operatorname{InAs},{ }^{5} \operatorname{InSb},{ }^{6}$ and $\mathrm{InN}^{7}$ and expanded their applications from LEDs ${ }^{8-10}$ and LSCs ${ }^{11}$ to sentinel lymph node mapping ${ }^{12}$ and neural photostimulation. ${ }^{13}$

Among the III-V semiconductors, AlSb has been less studied due to difficulties in the bulk crystal growth. ${ }^{14}$ It has an indirect and direct band gap of approximately 1.6 and $2.4 \mathrm{eV}$ at $300 \mathrm{~K}$ in the bulk form, respectively. ${ }^{15}$ To date, it was prepared only via physical and electrochemical routes, and particularly, its physical and technological properties were improved by incorporating with $\mathrm{GaSb}$ for near-infrared optoelectronic devices, ${ }^{16}$ quantum cascade lasers (QCLs) ${ }^{17}$ and high-speed field-effect transistors (FETs). ${ }^{18}$ Historically, polycrystalline $\mathrm{AlSb}$ was synthesized by high-temperature Czochralski and Bridgman methods, ${ }^{19}$ molecular beam epitaxy (MBE), ${ }^{17}$ and coevaporation of the elements. ${ }^{20}$ All of these costly methods require $\mathrm{H}_{2}$ at high temperatures to provide a reductive environment for AlSb crystal growth. Instead, the colloidal synthesis of QDs possesses the advantages of easy and low-cost material production in comparison with the above-mentioned physical methods and adaptability for solution-processable and large-scale device fabrication. But, to the best of our knowledge, there is no report in the literature for the colloidal synthesis of AlSb QDs to date.

The development of reliable and reproducible methods for synthesizing bright and tunable III-V QDs has been a major goal in materials chemistry research over the past decades. However, there are some challenges in the synthesis of these QDs. Strong tetrahedral coordinations in covalently bonded III-V compounds have slow crystallization in which higher reaction temperatures and longer growth time result in significant size broadening of the III-V QDs. Furthermore, using two separate monatomic precursors and the general lack of choice in appropriate precursors limit the control of colloid nucleation and growth. Specifically, for the synthesis of AlSb, the major challenge is the inappropriate reactivity of antimony precursors. $^{21-23}$ As $\mathrm{P}(\mathrm{TMS})_{3}$ was extensively used for the synthesis of $\mathrm{InP}, \mathrm{Sb}(\mathrm{TMS})_{3}$ is expected to lead AlSb QDs formation, but, due to the increase in the atomic radii from $\mathrm{P}$ to $\mathrm{Sb}$, it has yielded little success in the synthesis of InSb. ${ }^{21,23}$ Other than that, using $\mathrm{SbH}_{3}{ }^{24}$ as the $\mathrm{Sb}$ precursor is limited due to its high toxicity and reactivity. Alternatively, $\mathrm{Sb}[\mathrm{N}(\mathrm{Si}-$ $\left.\left.(\mathrm{Me})_{3}\right)_{2}\right]_{3}$ showed a potential for the synthesis of antimonybased QDs ${ }^{6,25}$ due to their polar M-N bonds, which can be used in the syntheses of metal and metalloid nanoparticles. ${ }^{26}$ Because of that, in our AlSb synthesis, we used $\mathrm{Sb}[\mathrm{N}(\mathrm{Si}-$ $\left.\left.(\mathrm{Me})_{3}\right)_{2}\right]_{3}$ as the $\mathrm{Sb}$ precursor.

\section{RESULTS AND DISCUSSION}

To synthesize AlSb QDs, first, $\mathrm{Sb}\left[\mathrm{N}\left(\mathrm{Si}(\mathrm{Me})_{3}\right)_{2}\right]_{3}$ was produced by the metathesis reaction between $\mathrm{SbCl}_{3}$ and the strong base $\mathrm{LiN}\left(\mathrm{Si}\left(\mathrm{CH}_{3}\right)_{3}\right)_{2}$, as reported previously. ${ }^{6} \mathrm{LiN}(\mathrm{Si}$ $\left.\left(\mathrm{CH}_{3}\right)_{3}\right)_{2}$ is a strong nucleophilic base $(\mathrm{pKa} \sim 30)$ and reacts with protic reagents and solvents for the synthesis of various metals, metalloids, and alloy nanocrystals ${ }^{27}$ (Figure 1a).

Received: March 5, 2019

Revised: June 9, 2019

Published: June 10, 2019 
(a)

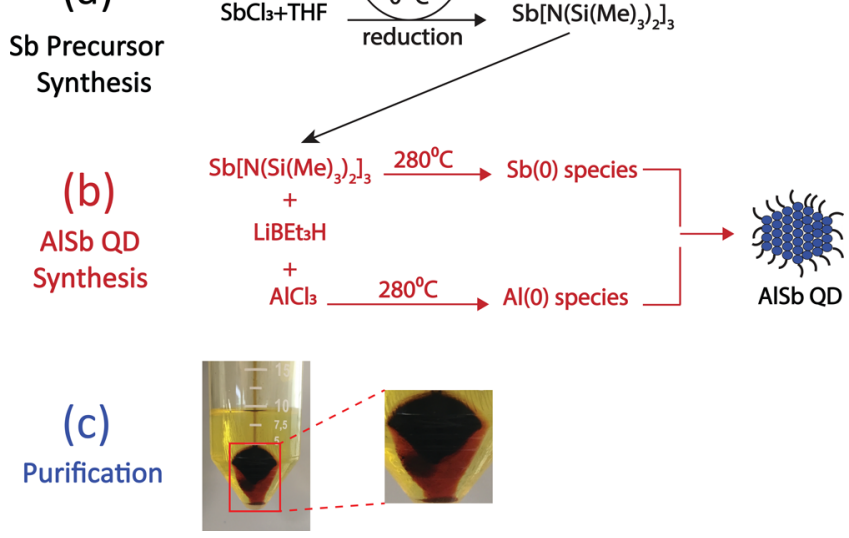

Figure 1. Colloidal synthesis steps of AlSb QDs. (a) Synthesis of antimony bis(trimethylsilyl)amide $\left(\mathrm{Sb}\left[\mathrm{N}\left(\mathrm{Si}(\mathrm{Me})_{3}\right)_{2}\right]_{3}\right)$ at $0{ }^{\circ} \mathrm{C}$ using $\mathrm{LiN}\left(\mathrm{Si}\left(\mathrm{CH}_{3}\right)_{3}\right)_{2}$ as the reduction agent and THF as the stabilizer. (b) Synthesis of colloidal AlSb QDs at $280{ }^{\circ} \mathrm{C}$ in the presence of superhydride. (c) Separation of AlSb QDs (brown) from impurities (black) after centrifugation. We observed that while the impurities do not have emission, QDs emit in blue color under UV. The centrifugation was repeated 10 times until no black precipitates were observed.

Adding THF as the coordinating solvent stabilizes the product due to its electron-donating capability. ${ }^{6}$ Then, we mixed $\mathrm{AlCl}_{3}$ and $\mathrm{Sb}\left[\mathrm{N}\left(\mathrm{Si}(\mathrm{Me})_{3}\right)_{2}\right]_{3}$ in the presence of superhydride $\left(\mathrm{LiEt}_{3} \mathrm{BH}\right)$ to form AlSb QDs (Figure $1 \mathrm{~b}$ ). $\mathrm{LiEt}_{3} \mathrm{BH}$ is a strong reducing agent, which can simultaneously reduce (coreduction) both $\mathrm{Al}$ and $\mathrm{Sb}$ precursors to form $\mathrm{Al}(0)$ and $\mathrm{Sb}(0)$ species at low temperatures, as reported for InSb. ${ }^{6}$ In the absence of superhydride, the resulting product contains metallic antimony, and at the reaction temperature lower than $200{ }^{\circ} \mathrm{C}$, the product invariably showed poor crystallinity. From these observations, the reaction mixture was heated up to $280{ }^{\circ} \mathrm{C}$ for $20 \mathrm{~min}$ to ensure crystallization. After the synthesis, a two-step purification process was performed to ensure the effective removal of unreacted precursors and excess ligands. For that, we first centrifuged the after-synthesis solution at a low temperature $\left(5{ }^{\circ} \mathrm{C}\right.$ ) (for several times) to separate the nonemitting black impurities from the emitting AlSb QDs (Figure 1c). Then, the QDs were collected from the supernatant by the common precipitation-dissolution method and finally dispersed in hexane for further characterization. The nanocolloids in the solution remained stable after 3 months.

To explore the size tunability of AlSb QDs, three aliquots were taken at different reaction times of 1,3 , and $20 \mathrm{~min}$ at $280{ }^{\circ} \mathrm{C}$. We see an absorption spectrum with a single shoulder that can be attributed to the first excitonic peak (Figure 2a). For these aliquots, excitonic transitions were observed from $3.32 \mathrm{eV}$ (for sample having $1 \mathrm{~min}$ reaction time) to $3.23 \mathrm{eV}$ (for sample having $20 \mathrm{~min}$ reaction time). As the QDs grow, the absorbance value increases, possibly due to the increase in the extinction coefficient like in InP QDs. ${ }^{28}$ Their band-edge PL peak is tuned from 455 to $485 \mathrm{~nm}$ (Figure $2 \mathrm{~b}, \mathrm{c}$ ). Then, for understanding the nature of optical transitions, we performed quantum mechanical calculations in an effective mass approximation. ${ }^{13,29}$ The effective exciton Bohr radius and Rydberg energy were calculated to be $5.67 \mathrm{~nm}$ and $11.33 \mathrm{meV}$, respectively. We found out that the optical transitions take place over the AlSb direct band gap at the $\Gamma$-valley under a strong confinement regime.

Moreover, the absolute fluorescence QY of these QDs in hexane was measured with an integrating sphere (Figure 2c).
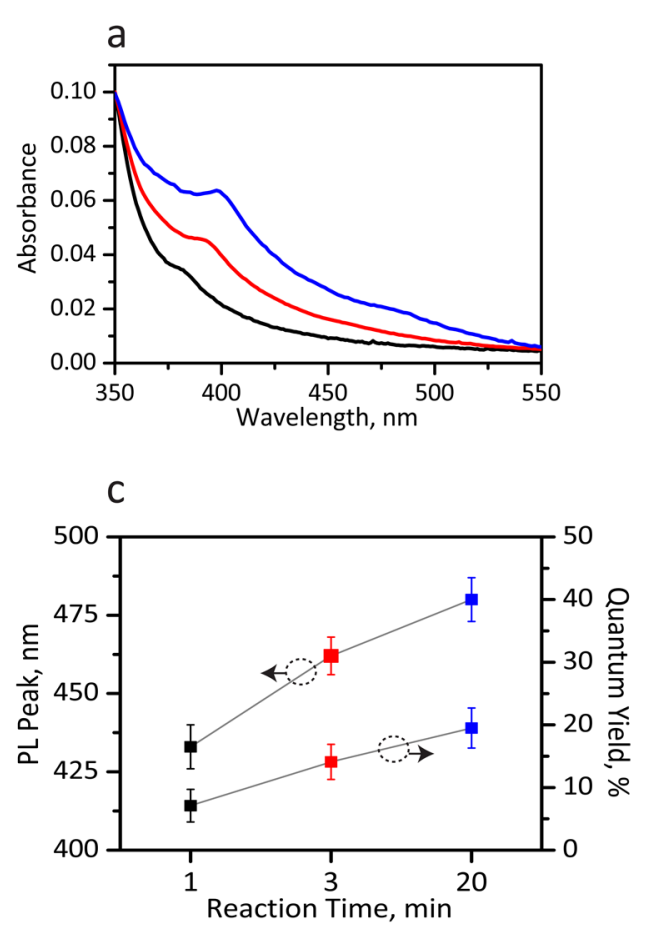
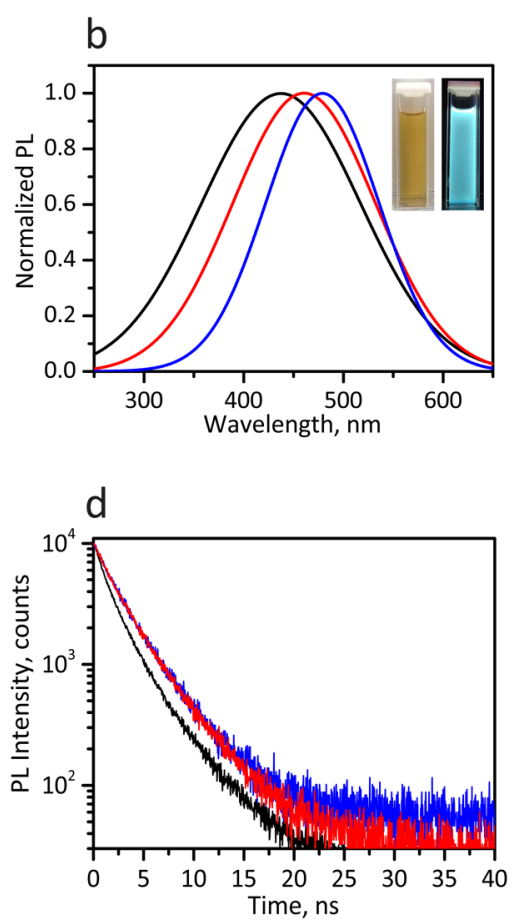

Figure 2. Optical characterization of colloidal AlSb QDs dispersed in hexane. (a) Absorbance, (b) normalized PL (left inset: photograph of the QD solution under ambient light; right inset: photograph of the QD solution under UV), (c) PL peak position and absolute QY values, and (d) timeresolved PL decays of different-sized colloidal AlSb QDs having 1 min (black), 3 min (red), and 20 min (violet) reaction times. 
Table 1. Time-Resolved PL Decay Analysis of Different-Sized Colloidal AlSb QDs

\begin{tabular}{cllrr} 
reaction time $(\mathrm{min})$ & $A_{1}(\mathrm{kCnts})$ & $A_{2}(\mathrm{kCnts})$ & $\tau_{1}(\mathrm{~ns})$ & $\tau_{2}(\mathrm{~ns})$ \\
1 & $10.5 \pm 0.12$ & $4.24 \pm 0.13$ & $1.31 \pm 0.01$ & $3.65 \pm 0.04$ \\
3 & $1.57 \pm 0.09$ & $2.98 \pm 0.12$ & $4.29 \pm 0.05$ & $1.99 \pm 0.01$ \\
20 & $0.58 \pm 0.08$ & $1.48 \pm 0.07$ & $4.41 \pm 0.13$ & $2.76 \pm 0.16$ \\
\hline
\end{tabular}

AlSb QDs emit with a high-fluorescence QY up to $18 \%$ coming from the band-edge emission. The previous studies also reported similar $\mathrm{QY}$ levels for core $\mathrm{InP},{ }^{4}$ InAs, ${ }^{5} \mathrm{InSb}^{6},{ }^{6}$ and $\mathrm{InN}^{7}$ at longer wavelengths. In comparison, the $\mathrm{AlSb}$ core shows the highest $\mathrm{QY}$ in the blue spectral region. Their PL dynamics were also studied using the general methodology of time-resolved photoluminescence via a time-correlated singlephoton counting (Figure 2d). PL decays were fitted by two exponential decays, ${ }^{28}$ and the average lifetime was calculated from an amplitude weighted mean (eq 1), which shows faster decay for large QDs (having longer reaction time) compared with small QDs (having shorter reaction time) (Table 1), as reported for indium-based QDs. ${ }^{28}$

$$
\tau_{\text {avg }}=\frac{A_{1} \tau_{1}^{2}+A_{2} \tau_{2}^{2}}{A_{1} \tau_{1}+A_{2} \tau_{2}}
$$

The HR-TEM imaging and image analysis revealed the QDs of AlSb with an average diameter of $9.7 \pm 0.8 \mathrm{~nm}$ (Figure $3 \mathrm{a}$ ).
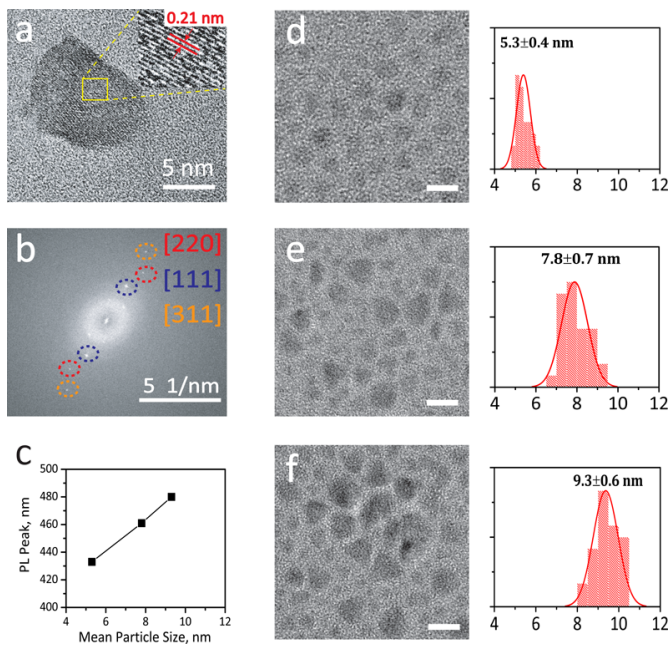

Figure 3. TEM analysis of the colloidal AlSb QDs. (a) Bright-field HR-TEM image. (Inset: high-resolution TEM image of a single AlSb QD). (b) Spatial frequencies in the FFT corresponding to the HRTEM image components arising from the AlSb crystallographic planes. (c) PL peak change as a function of the mean particle size. (d-f) TEM images (middle panel) and particle size distribution (right panel) of the colloidal AlSb QDs having reaction times of (d) 1 min, (e) $3 \mathrm{~min}$, and (f) $20 \mathrm{~min}$. (scale bars: $10 \mathrm{~nm}$ )

The interplanar distance of $0.21 \mathrm{~nm}$ corresponds to the (220) plane of the cubic AlSb structure (Figure 3a, inset). Analysis of the pseudodiffraction pattern (spatial frequency distribution by FFT) from one of the crystalline AlSb QDs confirmed the presence of (311), (220), and (111) planes of the cubic AlSb crystal structure, which is related to the $d$ spacings of 1.2, 3.2, and $3.6 \mathrm{~nm}$ (PDF 00-006-0233) (Figure 3b). The same pseudodiffraction pattern analysis has been performed in the characterization of other types of QDs as well. ${ }^{13}$ For statistics, conventional bright-field imaging of QDs was also performed, and the mean particle sizes of 5.5, 7.3, and $9.7 \mathrm{~nm}$ (Figure
$3 c)$ were determined for samples having growth times of $1 \mathrm{~min}$ (Figure 3d), $3 \mathrm{~min}$ (Figure 3e), and $20 \mathrm{~min}$ (Figure 3f), respectively.

To identify the phase of the synthesized QDs, XRD and EDS characterizations were recorded, which suggest the formation of nanocrystalline $\mathrm{QDs}$ of $\mathrm{AlSb}$ in the cubic phase (Figure 4a). Since aluminum (bond energy, Al-O $512 \mathrm{~kJ}$
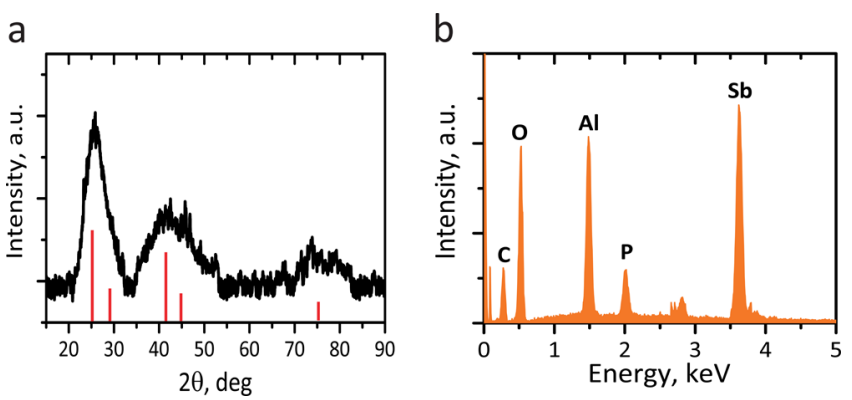

Figure 4. Structural analysis of the colloidal AlSb QDs. (a) XRD pattern (the vertical lines at the bottom are the corresponding positions and intensities of X-ray reflections for the bulk AlSb (PDF 00-006-0233)). (b) EDS spectrum of the colloidal AlSb QDs (origin of other peaks: $\mathrm{C}$ and $\mathrm{O}$ from the ligand (oleic acid), $\mathrm{P}$ from the TOP)).

$\left.\mathrm{mol}^{-1}\right)^{30}$ has a highly oxophilic nature, AlSb is prone to oxidation, but we did not observe any crystalline alumina peaks at $35,44,53$, and 58 . We were able to confirm the main features of the diffraction profile of our sample repeatedly with multiple data sets and by using two different diffractometers. The broad peaks at 25.1, 29.0, 41.6, 49.2, and 75.9 correspond to the (111), (200), (220), (311), and (422) crystal planes of the cubic AlSb, respectively, (PDF 00-006-0233). In the XRD pattern, the linewidth broadening and intensity strength observed are due to the drop-cast samples lacking a preferred orientation or periodic stacking, combined with the small size of the crystalline domain. Using Scherrer analysis, the average size of the crystalline domains was estimated to be $\sim 7 \mathrm{~nm}$. Calculation of the particle size with the modified Scherrer equation $(K=0.94)^{31}$ will lead to an effective diameter, which is smaller than the geometric diameter. Finally, energydispersive X-ray spectroscopy (EDS) analysis was performed to prove the elemental composition of the product (Figure 4b). It shows the stoichiometric compositions of $\mathrm{Al}$ and $\mathrm{Sb}$, within the experimental accuracy of EDS analysis. The higher amount of $\mathrm{Sb}$ can be attributed to the partial decomposition of the excess $\mathrm{Al}$ chloride at the QD surface.

\section{CONCLUSIONS}

In conclusion, AlSb colloidal QDs were synthesized by reacting $\mathrm{AlCl}_{3}$ and $\mathrm{Sb}\left[\mathrm{N}\left(\mathrm{Si}(\mathrm{Me})_{3}\right)_{2}\right]_{3}$ in the presence of superhydride. The QD reaction time was changed from 1 to $20 \mathrm{~min}$, which tuned the size range from 5.5 to $9.7 \mathrm{~nm}$. At the same time, while the quantum dots grow, a clearly observable first excitonic peak wavelength and photoluminescence red shift were obtained due to the quantum confinement effect. The 
photoluminescence in the blue spectral region with a high QY is also in agreement with the band-edge transition at the $\Gamma$ valley of AlSb. XRD, HR-TEM imaging, and analysis of the pseudodiffraction pattern confirmed the presence of cubic $\mathrm{AlSb}$ crystal planes. EDS analysis also indicates the stoichiometric elemental compositions. The toxic, heavy-metal-free content combined with bright emission shows a high potential for biological and optoelectronic applications.

\section{METHODS}

Materials. $\mathrm{LiN}\left(\mathrm{Si}\left(\mathrm{CH}_{3}\right)_{3}\right)_{2} \quad(97 \%), \mathrm{SbCl}_{3} \quad(>99 \%), \quad \mathrm{THF}$ ( $>99.5 \%), \mathrm{LiEt}_{3} \mathrm{BH}$ solution (in THF), OA (>90\%), TOP (97\%), and $\mathrm{AlCl}_{3}$ (99\%) were purchased from Sigma-Aldrich. Diethyl ether ( $>98 \%$ ) was purchased from Merck. OAM (>98\%) was purchased from Aldrich.

General Considerations. All reactions were run in a nitrogenfilled glovebox with an $\mathrm{O}_{2}$ level below $1 \mathrm{ppm}$ or using the standard Schlenk technique under an inert atmosphere or nitrogen. $\mathrm{LiEt}_{3} \mathrm{BH}$ solution is extremely air sensitive and needs to be handled carefully in glove-box.

Synthesis of $\mathrm{Sb}\left[\mathrm{N}\left(\mathrm{Si}(\mathrm{Me})_{3}\right)_{2}\right]_{3}$. It was synthesized by the metathesis reaction between $\mathrm{SbCl}_{3}$ and $\mathrm{LiN}\left(\mathrm{Si}\left(\mathrm{CH}_{3}\right)_{3}\right)_{2}$. In short, $38 \mathrm{mmol} \mathrm{LiN}\left(\mathrm{Si}\left(\mathrm{CH}_{3}\right)_{3}\right)_{2}$ were dissolved in $80 \mathrm{~mL}$ of diethyl ether in the dark and cooled down to $0{ }^{\circ} \mathrm{C}$. Thirteen millimoles of $\mathrm{SbCl}_{3}$ dissolved in the mixture of $2 \mathrm{~mL}$ of THF and $8 \mathrm{~mL}$ of diethyl ether was slowly added to the main reaction flask. The resulting white turbid solution was stirred for $12 \mathrm{~h}$, centrifuged to remove $\mathrm{LiCl}$, and filtered through a $0.45 \mu \mathrm{m}$ PTFE filter. After the removal of the solvents under vacuum, the residue was dissolved in hexane, filtered through a $0.45 \mu \mathrm{m}$ PTFE filter again, and dried under vacuum. A yellow solid product was obtained after the complete removal of hexane. The reaction yield was $\sim 85 \%{ }^{6}{ }^{6}$ The product was dispersed in $22 \mathrm{~mL}$ of toluene $\left(0.5 \mathrm{mmol} \mathrm{ml}^{-1}\right)$ and stored under an inert atmosphere in the dark at $-40{ }^{\circ} \mathrm{C}$.

Synthesis of AISb QDs. First, $0.16 \mathrm{mmol} \mathrm{AlCl}_{3}, 300 \mu \mathrm{L}(0.16$ mmol) $\mathrm{Sb}\left[\mathrm{N}\left(\mathrm{Si}(\mathrm{Me})_{3}\right)_{2}\right]_{3}$, and $640 \mu \mathrm{L}$ TOP were mixed in the glovebox. Then, $1 \mathrm{~mL}$ of $1 \mathrm{M} \mathrm{LiEt}_{3} \mathrm{BH}$ solution and $2.4 \mathrm{~mL}$ of OAM were mixed and added into the main reaction flask. The color of the solution became dark immediately after the injection. The solution was heated to $280{ }^{\circ} \mathrm{C}$, kept for $20 \mathrm{~min}$, and then cooled down to room temperature. One milliliter of OA was added to the solution to neutralize the excessive superhydride and attach oleate ligands to the AlSb surface. Then, the resulting solution was centrifuged at $5{ }^{\circ} \mathrm{C}$ at $5000 \mathrm{rpm}$ for 10 times to separate QDs from the impurities. Finally, to collect the QDs, ethanol/acetone (1:1) was added to the QD solution, centrifuged at $9000 \mathrm{rpm}$, and dispersed in hexane for further characterization. Multiple-sized QDs were provided by taking three aliquots at different reaction times of 1,3 , and $20 \mathrm{~min}$.

Absorbance, PL. Absorbance and PL spectra of the QDs dispersed in hexane were measured by an Edinburgh Instruments FS5 spectrofluorometer in the range of $300-700 \mathrm{~nm}\left(\lambda_{\text {exc }}=275 \mathrm{~nm}\right)$.

QY Measurement. The absolute fluorescence QYs were measured with an integrating sphere on an Edinburgh Instruments FS5 spectrofluorometer.

Time-Resolved PL. PL decays were recorded by a PicoQuant MicroTime 100 time-resolved confocal fluorescence microscope. The QD solution was excited by an $8 \mathrm{~mW}$ picosecond diode laser $\left(\lambda_{\text {exc }}=\right.$ $375 \mathrm{~nm}$ ) pulsed at a $60 \mathrm{MHz}$ repetition rate.

TEM. High-resolution transmission electron microscopy (TEM) analysis was performed using a JEOL JEM-ARM200CFEG UHR microscope with a spherical aberration-corrected probe $(200 \mathrm{keV})$ and equipped with a Gatan Model 994 UltraScan 1000XP CCD camera. The conventional bright-field TEM images were collected using FEI Talos F200S by an accelerating voltage of $200 \mathrm{keV}$. The specimens for the TEM analysis were prepared by depositing directly a holey carbon film on a copper support grid. The HR-TEM images were processed by the software ImageJ (https://imagej.nih.gov/ij/) for particle size determination.
XRD and EDS. Multiple layers of QDs in hexane were drop-cast on a glass substrate, left to dry, and analyzed in a Bruker D2 Phaser Xray diffractometer with $\mathrm{Cu} \mathrm{K} \alpha$ radiation $\left(\lambda=1.541^{\circ} \mathrm{A}\right)$ and a Zeiss Ultra Plus field emission scanning electron microscope by an accelerating voltage of $12 \mathrm{keV}$.

\section{ASSOCIATED CONTENT}

\section{Supporting Information}

The Supporting Information is available free of charge on the ACS Publications website at DOI: 10.1021/acs.chemmater.9b00905.

Details of quantum mechanical calculations, variables used in the quantum mechanical calculations, and parameters of AlSb used in the quantum mechanical calculations (PDF)

\section{AUTHOR INFORMATION}

\section{Corresponding Author}

*E-mail: snizamoglu@ku.edu.tr.

ORCID

Houman Bahmani Jalali: 0000-0001-7212-9098

Sadra Sadeghi: 0000-0002-8569-1626

Mehmet Sahin: 0000-0002-9419-1711

Cleva W. Ow-Yang: 0000-0002-2909-0957

Sedat Nizamoglu: 0000-0003-0394-5790

Author Contributions

${ }^{\nabla}$ H.B.J. and S.S. contributed equally to the manuscript.

\section{Funding}

This project has received funding from the European Research Council (ERC) under the European Union's Horizon 2020 research and innovation programme (grant agreement no. 639846).

\section{Notes}

The authors declare no competing financial interest.

\section{ACKNOWLEDGMENTS}

We sincerely thank Dr. Wenyong Liu of the University of Chicago for his helpful discussions about the synthesis of $\mathrm{Sb}\left[\mathrm{N}\left(\mathrm{Si}(\mathrm{Me})_{3}\right)_{2}\right]_{3}$. We thank KUYTAM (Koç University Surface Science and Technology Center) for the EDS and $\mathrm{XRD}$ infrastructures.

\section{ABBREVIATIONS}

$\mathrm{QD}$, quantum dot

$\mathrm{Cd}$, cadmium

$\mathrm{Pb}$, lead

InP, indium phosphide

InAs, indium arsenide

$\mathrm{InSb}$, indium antimony

$\mathrm{InN}$, indium nitride

LED, light-emitting diode

LSC, luminescent solar concentrator

$\mathrm{AlSb}$, aluminum antimonide

$\mathrm{GaSb}$, gallium antimony

QCL, quantum cascade laser

FETs, field-effect transistors

MBE, molecular beam epitaxy

$\mathrm{H}_{2}$, hydrogen gas

$\mathrm{P}(\mathrm{TMS})_{3}$, phosphine tris (trimethylsilyl)

$\mathrm{Sb}$ (TMS) $)_{3}$, antimony tris(trimethylsilyl)

$\mathrm{Sb}\left[\mathrm{NMe}_{2}\right]_{3}$, antimony tris(dimethylamido) 
$\mathrm{Sb}\left[\mathrm{N}\left(\mathrm{Si}(\mathrm{Me})_{3}\right)_{2}\right]_{3}$, antimony bis(trimethylsilyl)amide

$\mathrm{SbH}_{3}$, stibine gas

$\mathrm{SbCl}_{3}$, antimony trichloride

$\mathrm{Li}\left[\mathrm{N}\left(\mathrm{Si}\left(\mathrm{CH}_{3}\right)_{3}\right)_{2}\right]$, lithium bis(trimethylsilyl)amide

THF, tetrahydrofuran

$\mathrm{AlCl}_{3}$, aluminum chloride

$\mathrm{eV}$, electron volt

PL, photoluminescent

QY, quantum yield

HR-TEM, high-resolution transmission electron microscopy

FFT, fast Fourier transform

$\mathrm{XRD}, \mathrm{X}$-ray diffraction

EDS, energy-dispersive $\mathrm{X}$-ray spectroscopy

$\mathrm{LiCl}$, lithium chloride

TOP, trioctylphosphine

OAM, oleylamine

OA, oleic acid

\section{REFERENCES}

(1) Kagan, C. R.; Lifshitz, E.; Sargent, E. H.; Talapin, D. V. Building devices from colloidal quantum dots. Science 2016, 353, aac5523.

(2) Sadeghi, S.; Kumar, B. G.; Melikov, R.; Aria, M. M.; Jalali, H. B.; Nizamoglu, S. Quantum dot white LEDs with high luminous efficiency. Optica 2018, 5, 793-802.

(3) Soenen, S. J.; Rivera-Gil, P.; Montenegro, J.-M.; Parak, W. J.; De Smedt, S. C.; Braeckmans, K. Cellular toxicity of inorganic nanoparticles: Common aspects and guidelines for improved nanotoxicity evaluation. Nano Today 2011, 6, 446-465.

(4) Xu, S.; Ziegler, J.; Nann, T. Rapid synthesis of highly luminescent $\mathrm{InP}$ and $\mathrm{InP} / \mathrm{ZnS}$ nanocrystals. J. Mater. Chem. 2008, $18,2653-2656$.

(5) Franke, D.; Harris, D. K.; Chen, O.; Bruns, O. T.; Carr, J. A.; Wilson, M. W. B.; Bawendi, M. G. Continuous injection synthesis of indium arsenide quantum dots emissive in the short-wavelength infrared. Nat. Commun. 2016, 7, 12749.

(6) Liu, W.; Chang, A. Y.; Schaller, R. D.; Talapin, D. V. Colloidal InSb Nanocrystals. J. Am. Chem. Soc. 2012, 134, $20258-20261$.

(7) Chen, Z.; Li, Y.; Cao, C.; Zhao, S.; Fathololoumi, S.; Mi, Z.; Xu, $X$. Large-Scale Cubic InN Nanocrystals by a Combined Solution- and Vapor-Phase Method under Silica Confinement. J. Am. Chem. Soc. 2012, 134, 780-783.

(8) Karatum, O.; Jalali, H. B.; Sadeghi, S.; Melikov, R.; Srivastava, S. B.; Nizamoglu, S. Light-Emitting Devices Based on Type-II InP/ZnO Quantum Dots. ACS Photonics 2019, 6, 939-946.

(9) Demir, H. V.; Nizamoglu, S.; Erdem, T.; Mutlugun, E.; Gaponik, N.; Eychmüller, A. Quantum dot integrated LEDs using photonic and excitonic color conversion. Nano Today 2011, 6, 632-647.

(10) Kumar, B. G.; Sadeghi, S.; Melikov, R.; Aria, M. M.; Jalali, H. B.; Ow-Yang, C. W.; Nizamoglu, S. Structural control of InP/ZnS core/shell quantum dots enables high-quality white LEDs. Nanotechnology 2018, 29, 345605.

(11) Sadeghi, S.; Bahmani Jalali, H.; Melikov, R.; Ganesh Kumar, B.; Mohammadi Aria, M.; Ow-Yang, C. W.; Nizamoglu, S. Stokes-ShiftEngineered Indium Phosphide Quantum Dots for Efficient Luminescent Solar Concentrators. ACS Appl. Mater. Interfaces 2018, 10, 12975-12982.

(12) Kim, S.-W.; Zimmer, J. P.; Ohnishi, S.; Tracy, J. B.; Frangioni, J. V.; Bawendi, M. G. Engineering InAs $\mathrm{P}_{\mathrm{x}} \mathrm{P}_{1-\mathrm{x}} / \mathrm{InP} / \mathrm{ZnSe}$ III-V Alloyed Core/Shell Quantum Dots for the Near-Infrared. J. Am. Chem. Soc. 2005, 127, 10526-10532.

(13) Bahmani Jalali, H.; Mohammadi Aria, M.; Dikbas, U. M.; Sadeghi, S.; Ganesh Kumar, B.; Sahin, M.; Kavakli, I. H.; Ow-Yang, C. W.; Nizamoglu, S. Effective Neural Photostimulation Using IndiumBased Type-II Quantum Dots. ACS Nano 2018, 12, 8104-8114.

(14) Adachi, S. Optical constants of crystalline and amorphous semiconductors: numerical data and graphical information; Springer Science \& Business Media: 1999.
(15) Bornstein, L. Numerical data and functional relationships in science and technology. Semiconductors: Physics of Group IV Elements and III-V Compounds, New Series; 1982, 17.

(16) Schwartz, G. P.; Gualtieri, G. J.; Sunder, W. A.; Farrow, L. A. Light scattering from quantum confined and interface optical vibrational modes in strained-layer $\mathrm{GaSb} / \mathrm{AlSb}$ superlattices. Phys. Rev. B 1987, 36, 4868-4877.

(17) Barate, D.; Teissier, R.; Wang, Y.; Baranov, A. N. Short wavelength intersubband emission from InAs/AlSb quantum cascade structures. Appl. Phys. Lett. 2005, 87, No. 051103.

(18) Glaser, E. R.; Kennedy, T. A.; Bennett, B. R.; Shanabrook, B. V. Strong emission from As monolayers in AlSb. Phys. Rev. B 1999, 59, 2240-2244.

(19) Linnebach, R.; Benz, K. W. Bridgman growth of AlSb. J. Cryst. Growth 1981, 53, 579-585.

(20) Johnson, J. E. Aluminum Antimonide Thin Films by Coevaporation of the Elements. J. Appl. Phys. 1965, 36, 3193-3195.

(21) Evans, C. M.; Castro, S. L.; Worman, J. J.; Raffaelle, R. P. Synthesis and Use of Tris(trimethylsilyl)antimony for the Preparation of InSb Quantum Dots. Chem. Mater. 2008, 20, 5727-5730.

(22) Kim, S.-W.; Sujith, S.; Lee, B. Y. InAs $\mathrm{Sb}_{1-\mathrm{x}}$ alloy nanocrystals for use in the near infrared. Chem. Commun. 2006, 4811-4813.

(23) Tamang, S.; Kim, K.; Choi, H.; Kim, Y.; Jeong, S. Synthesis of colloidal InSb nanocrystals via in situ activation of $\mathrm{InCl}_{3}$. Dalton Trans. 2015, 44, 16923-16928.

(24) Maurice, A.; Haro, M. L.; Hyot, B.; Reiss, P. Synthesis of Colloidal Indium Antimonide Nanocrystals Using Stibine. Part. Part. Syst. Charact. 2013, 30, 828-831.

(25) Yarema, M.; Kovalenko, M. V. Colloidal synthesis of InSb nanocrystals with controlled polymorphism using indium and antimony amides. Chem. Mater. 2013, 25, 1788-1792.

(26) Yarema, M.; Caputo, R.; Kovalenko, M. V. Precision synthesis of colloidal inorganic nanocrystals using metal and metalloid amides. Nanoscale 2013, 5, 8398-8410.

(27) He, M.; Protesescu, L.; Caputo, R.; Krumeich, F.; Kovalenko, M. V. A general synthesis strategy for monodisperse metallic and metalloid nanoparticles (In, Ga, Bi, Sb, $\mathrm{Zn}, \mathrm{Cu}, \mathrm{Sn}$, and their alloys) via in situ formed metal long-chain amides. Chem. Mater. 2015, 27, 635-647.

(28) Bahmani Jalali, H.; Melikov, R.; Sadeghi, S.; Nizamoglu, S. Excitonic energy transfer within $\mathrm{InP} / \mathrm{ZnS}$ quantum dot langmuirblodgett assemblies. J. Phys. Chem. C 2018, 122, 11616-11622.

(29) Şahin, M.; Nizamoglu, S.; Kavruk, A. E.; Demir, H. V. Selfconsistent computation of electronic and optical properties of a single exciton in a spherical quantum dot via matrix diagonalization method. J. Appl. Phys. 2009, 106, No. 043704.

(30) Dean, J. A. Lange's handbook of chemistry; McGraw-Hill, Inc.: New york, London, 1999.

(31) Langford, J. I.; Wilson, A. J. C. Scherrer after sixty years: a survey and some new results in the determination of crystallite size. J. Appl. Crystallogr. 1978, 11, 102-113. 